\title{
Bayesian Learning of Models for Estimating Uncertainty in Alert Systems: Application to Air Traffic Conflict Avoidance
}

\author{
V. Schetinin ${ }^{\mathrm{a}, *}$, L. Jakaite ${ }^{\mathrm{a}}$ and W. Krzanowski ${ }^{\mathrm{b}}$ a School of Computer Science and Technology, University of \\ Bedfordshire, $U K^{\mathrm{b}}$ College of Engineering, Mathematics and Physical Sciences, University of Exeter, UK
}

\begin{abstract}
Alert systems detect critical events which can happen in the short term. Uncertainties in data and in the models used for detection cause alert errors. In the case of air traffic control systems such as Short-Term Conflict Alert (STCA), uncertainty increases errors in alerts of separation loss. Statistical methods that are based on analytical assumptions can provide biased estimates of uncertainties. More accurate analysis can be achieved by using Bayesian Model Averaging, which provides estimates of the posterior probability distribution of a prediction. We propose a new approach to estimate the prediction uncertainty, which is based on observations that the uncertainty can be quantified by variance of predicted outcomes. In our approach, predictions for which variances of posterior probabilities are above a given threshold are assigned to be uncertain. To verify our approach we calculate a probability of alert based on the extrapolation of closest point of approach. Using Heathrow airport flight data we found that alerts are often generated under different conditions, variations in which lead to alert detection errors. Achieving $82.1 \%$ accuracy of modelling the STCA system, which is a necessary condition for evaluating the uncertainty in prediction, we found that the proposed method is capable of reducing the uncertain component. Comparison with a bootstrap aggregation method has demonstrated a significant reduction of uncertainty in predictions. Realistic estimates of uncertainties will open up new approaches to improving the performance of alert systems.
\end{abstract}

Keywords. Alert systems, Uncertainty, Air traffic control, Bayesian Model Averaging, Monte Carlo methods

\section{Introduction}

Alert systems aim to predict patterns of critical events which can happen in the short term. The systems are designed to predict events which must be avoided under specified conditions on given data. In alert systems the "ground truth" is unavailable and so has to be estimated within a simulated environment. This is ideally designed to realistically handle combinations of uncertainty factors involved in retrospective events, as well as in manoeuvring intents of pilots attempting to resolve a problem. The models are expected to provide plausible prediction of alert events. In safety-critical applications, such as transport systems [1|2]3], air traffic control [45] using simulation [6] and on-board Traffic Alert and Collision Avoidance Systems (TCAS) [7], civil engineering [ [8|9|10], and power plants [11/12 13], the estimation of accuracy suffers from uncertainties existing in both the models and the data which are used for predictions.

Some alerts can be predicted less certainly than others in terms of intervals of predicted probabilities, see e.g. [1|14]. In this light, prediction intervals reflect confidence of estimates within the probabilistic framework [15], providing important information about possible risks in the form of posterior predictive distributions.

In air-traffic control, uncertainties in the data and models affect predictions of the future positions of aircraft and increase the errors of detecting areas of a possible conflict [16]. Errors of false positive type are defined as false alarms or nuisance, whilst errors of false negative type are defined as late or missed alerts [17].

The uncertainty affects the accuracy of predicting the future positions of aircraft and increases errors in alerts of separation loss [ [18 $19 \mid 20]$. Short-Term Conflict Alert (STCA) systems are used in airports, that have complex and intensive air traffic, to warn operators and pilots when the distance between two aircraft is critically short in a given zone. Alerts generated by the STCA system warn about a possible conflict that is defined as loss of safe separation between two aircraft, see e.g. [21|22]. For prediction of possible conflicts, STCA systems use radar data provided by air traffic service. Distance and time to the Closest Point of Approach (CPA) between the aircraft are the main factors 
of collision risk, as defined in the pioneering research [23]. Factors such as wind, weather conditions, aircraft flight characteristics, unavoidable imprecision in operations and manoeuvres, as well as impression of radar readings increase uncertainty in air traffic data, see e.g. [24|25|26].

In an intensive air traffic environment, it is critically important to detect conflict alerts with the maximum accuracy and reliability. Analysis of uncertainty factors, which affect the detection accuracy, therefore will bring new insights into the problem [27|28].

Uncertainty in aircraft conflict detection can be formulated within a probabilistic framework which has been shown to be capable of improving the operational performance, as described in [29 30]. Other approaches based on Monte Carlo simulation have been considered in [31|32].

The uncertainty in a prediction can be quite large when the data sets available for building the models are small. To avoid this problem, it has been suggested that the performance should be reported in the form of a Bayesian confidence interval obtained by a method which provides conservative measures of the uncertainty [33]. Bayesian Model Averaging (BMA) methodology in theory provides the most accurate estimates of the predictive posterior distribution required for calculating prediction intervals. BMA has been made computationally feasible by using Markov Chain Monte Carlo (MCMC) approximations [34].

Bayesian inference has been used for the learning of flight trajectory patterns from real air traffic data [35 6 36]. The Bayesian MCMC method has been used to build a model of the STCA system from Heathrow air-traffic data, in order to analyse the uncertainty and determine factors that affect predictions [37]. Although the described method has demonstrated promising results in modelling the STCA system, patterns of predicted outcomes have not yet been estimated in terms of uncertainty. The analysis of such patterns is critically required in order to find areas in which STCA performance can be improved [17].

We observed that the Bayesian models can detect some patterns at a high uncertainty in the posterior predictive distribution. This happens because a large number of models generate contradictory outputs as a result of detecting two patterns, alert or normal. We can thus analyse models that vote for incorrect decisions in order to find factors that cause the uncertainty. Making patterns of interest transparent by using Decision Tree (DT) models, Bayesian analysis provides new insights into areas of potential improvements of prediction performance, as described in our previous work [38 39 40].

In this paper we describe a new Bayesian approach to modelling alert systems on given data in order to (i) analyse the posterior predictive probability distribution of alert patterns and (ii) provide insights into the uncertainty of alert events. As outlined above, alert systems generate data which do not contain "ground truth", and so modelling of the STCA system is required in order to identify patterns which are uncertain. Transparent modelling of an alert system therefore will bring insights into the uncertainty and conditions under which the uncertainty is raised.

In such circumstances, we assume that the uncertainty in alert patterns is characterised by the variance of the posterior predictive probability density. Predictions with a variance above a given threshold are identified as uncertain, and so the number of such predictions is important for evaluating the uncertainty of an alert system. In our experiments the proposed method demonstrated a substantial reduction in the number of uncertain predictions by comparison with the bootstrap aggregation described in [41].

For the research in this paper, flight data were made available by the UK National Air Traffic Services [42]. The proposed method will be used on these data to demonstrate the ability (i) to recognise both certain and uncertain predictions, and (ii) to provide a significantly larger number of certain predictions by comparison with the bootstrap aggregation. Reliable analysis of patterns which increase probabilities of errors is critically important for finding new insights into areas in which alert systems can be improved when their performance cannot be straightforwardly estimated.

The rest of the paper is organised as follows. Section 2 provides related work on air-traffic control. Section 3 introduces the STCA problem and describes the flight data that are used in our experiments. The simulation of alert probabilities is outlined in Section 4 The following Section 5 describes the methodology of Bayesian learning of DT models. The experiments with the proposed Bayesian method are described in Section 6, and the results are discussed in Section 7 Conclusions are presented in Section 8. Appendix A provides the geometrical extrapolation of the CPA, which is used for estimating alert probabilities.

\section{Related Work}

National authorities specify vertical and horizontal separation standards to maintain the safe navigation 
of aircraft in controlled zones. These standards ensure safe separation from the ground, other aircraft and from protected airspace [43]. When two aircraft in an airport environment are on a potential collision course, a conflict event occurs and the controller and pilots communicate a resolution action based on flight data. To insure that the minimum separation between the aircraft is maintained, the aircraft trajectory is predicted so as to avoid the conflict zone. In this section we outline work related to prediction of conflict events.

\subsection{Linear extrapolation in conflict alert and collision avoidance systems}

In [27], the influence of radar surveillance performance on conflict detection and separation distances has been analysed by modelling a conflict alert system. It has been reported that reliable assessment of the distances requires an advanced model which includes not only radar monitoring but also weather conditions under different failure scenarios.

Prediction of an aircrafts position in the future is based on extrapolation using the aircrafts flight data. The algorithm decides whether the aircraft pair will be in a critical zone within the time of arriving at the CPA. Based on such prediction, the TCAS [7] has significantly reduced the risk of collision [44[45]. There is a concern that false alarms decrease trust in the system and lead to ignorance of alerts [17].

Geometric-based predictions may be not sufficiently accurate when an aircraft does not behave as expected in the immediate future [46]. The uncertainty in a prediction is managed by increasing the minimum distance and threshold time to the CPA at which an alert will be detected. The alerts detected by using such a linear prediction model have to be adjusted so as to satisfy a trade-off between false and missed alert rates. It has been concluded that the probabilistic approach directly determines the required balance.

\subsection{Modelling with adjustable parameters}

A Gaussian model is assumed, reflecting the uncertainty along aircraft flight paths [27]. The model parameters are defined by an expected aircraft position and a variance determining a $95 \%$ position in the horizontal plane. Having adjusted the model parameters, a region is estimated for a given minimum separation, where a conflict is detected at a certain confidence interval.
A probabilistic conflict detection system presented in [5] assumes a model simulating the errors of trajectory prediction, which is used to minimise missed and false alerts. In every conflict detection cycle, two trajectories are simulated for each aircraft: one trajectory is a baseline and the other, which contains prediction errors, is used for simulating conflict detection and resolution. In every conflict detection cycle, the baseline is used to determine whether the conflict detection is an error or not, and thus the performance in terms of false rates is estimated at a given level of prediction uncertainty.

Monte Carlo simulation of air traffic control has been presented as a realistic approach for risk assessment [31]. The simulation is based on models which represent interactions between components of a system. Assessment of the risk of a conflict between the aircraft is based on the results of Monte Carlo simulations. In practice, when limited flight data are available, analysis of credibility intervals is required in order to realistically evaluate risks.

\subsection{Probabilistic modelling of air traffic}

Aircraft trajectory models, developed for evaluating the performance of air traffic alert systems, are limited in their ability to represent flight data [6]36]. The proposed methodology is based on a dynamic Bayesian network framework described in [47]. Experiments have demonstrated the efficiency of Bayesian models for evaluating risks of collisions.

The rigorous analysis of traffic control systems requires an accurate model of aircraft behaviour [6 36]. The feasibility of using a Markov decision process for analysis of an air-traffic alert system has been investigated in [26]. The different approaches for learning of traffic models from recorded flight data are evaluated. It has been found that one approach, which is based on prior trajectory patterns, performs well on simulated data, but it has difficulty with real-world data. The other approach uses Bayesian inference techniques to learn parameters of the traffic model employing a Markov model. This approach is made computationally feasible with MCMC methods. The Bayesian models have been found to better represent the observed data.

An important consideration in air traffic alert systems is how to evaluate uncertainty. Many collision avoidance systems use point estimates of the state instead of the full posterior state distribution. Decision theoretical methods have been applied to collision avoidance, but the importance of state uncertainty has 
required further investigation. A computationally efficient framework has been proposed in [20] for evaluating the uncertainty. Examination of Monte Carlo simulations has demonstrated that the estimates of state uncertainty can significantly enhance safety of collision avoidance systems.

Advanced algorithmic techniques have been developed for solving problems, which are caused by multiple sources of uncertainties in flight data, for collision avoidance [45]. These techniques employ probabilistic models capable of representing the uncertainty sources to optimise collision avoidance systems. Experiments on recorded flight data have confirmed a significant improvement in safety of collision avoidance systems.

Deterministic models of collision avoidance are not able to provide reliable estimates of collision risks. Probabilistic models are capable of representing the various sources of uncertainty. A model described in [48] has been developed within a probabilistic framework to handle the uncertainty in collision avoidance.

\subsection{Ensemble methods}

Ensemble methods, known from the literature also as bootstrap aggregation [4950] and bagging predictors [4151], aim to approximate the posterior predictive distribution by sampling from data given for building the models. The ensemble methods significantly improve the accuracy of prediction under certain conditions. A bagging method known as Random Forests (RF) [52] has provided substantial gains in accuracy, using such models as classification and regression trees.

The ensemble methods have improved the prediction accuracy although estimation of predictive distributions requires additional efforts to achieve conditions under which the predictions are asymptotically normal, and the confidence intervals can accompany predictions [53]. The variability of predictions made by the bagging methods and RF has been also estimated in the form of standard errors [14]. In [54], the uncertainty has been defined as the variation in the ensemble of models, which quantifies the "disagreement" between the model outcomes.

\subsection{Bayesian averaging over DT models}

The use of DT models within the Bayesian learning framework makes probabilistic inference transparent and capable of providing insights into factors that cause uncertainty in predictions [55|56|57]. DT models are defined as hierarchical structures of splitting and terminal nodes which recursively split data [58 59]. Tree-like models have been efficient for failure analysis and outcome prediction in engineering applications, see e.g. [6061].

The Bayesian method represents a prediction model as a Markov chain having transition states. The current state is dependent on the previous state. Under certain conditions the Markov chain achieves stationarity. These properties permit the generation of a large sample of model parameters, which is required to achieve the accurate approximation of predictive posterior density. By contrast, RF is based on the random subsampling from data and on random selection of predictors. This strategy can simulate the uncertainty in the data, but not in the model parameters, as we discussed in [62 63].

\section{STCA Problem and Data}

Air traffic control systems are primarily based on geometric extrapolation of the minimal distance and arrival time at the CPA for an aircraft pair [46 44 [5]. In our study we use this method to map the distances and times, which were estimated from the given flight data, onto alert probabilities that are required for evaluating the prediction accuracy.

\subsection{Representation of flight data}

The primary flight data are received from radar included in airport traffic control system. The received information is updated in each radar cycle. Flight data include radar positions of aircraft in the 3-dimension system of coordinates $x, y$ and $z$. The coordinates $x$ and $y$ define the position of an aircraft in the $x y$ lateral plane. The coordinates $x$ and $y$ are relative to the radar position, and the coordinate $z$ is altitude.

Fig. 1 shows the traces of an aircraft pair in coordinates $x, y$, and $z$. The alert cycles here are marked by the filled (in Red) circles, while the normal cycles are shown blank. The starting positions of the aircraft are indicated by the numbers 1 and 2 .

We can see that after the 20th radar cycle the system detects a series of 6 alarm cycles. Having been alerted, the pilot of aircraft 2 has undertaken an immediate manoeuvre to avoid a dangerous loss of separation with aircraft 1 . During the alert cycles, the distance between the aircraft critically decreased from 2500 to 90 . The projections of the aircraft trajectories (in Grey) in the $x y$ plane show that the aircraft ap- 


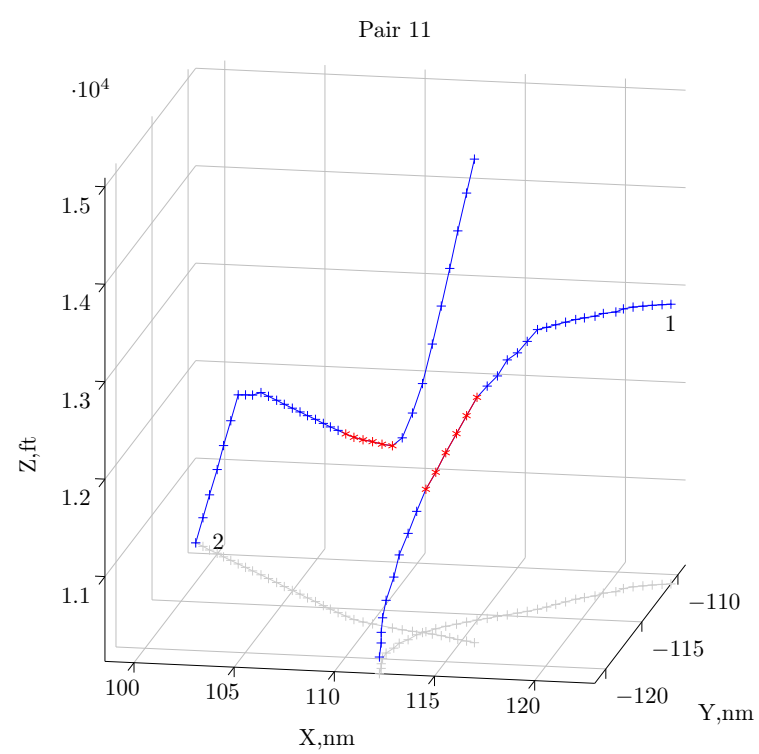

Figure 1. A radar track with alert cycles (in Red). Start positions of aircraft are denoted by the numbers 1 and 2 .

proached each other at least 5 cycles before the first alert. This means that an earlier alert would enable pilots and operators to plan a more safe and efficient manoeuvre.

In our experiments we used the flight data that represent traces of aircraft pairs with detected alerts. Each trace includes a sequence of radar cycles as described above. A cycle in the sequence represents the movement of the aircraft pair and can be either normal or alert. These data are selected for modelling the STCA system within the Bayesian framework in order to estimate the accuracy and uncertainty of alert detection.

For modelling the following predictor variables were extracted from the data: (i) the distances $d_{x}, d_{y}$, and $d_{z}$ between aircraft 1 and 2 along the coordinates $x, y$, and $z$, respectively, and (ii) the velocities $V_{x}, V_{y}$ and $V_{z}$ of the aircraft.

The distance between aircraft 1 and 2 is important for alert detection in the airport environment when aircraft change positions in coordinates $x, y$ and $z$ during landing or taking off, so that $d=\sqrt{\left(d_{x}^{2}+d_{y}^{2}\right) s^{2}+d_{z}^{2}}$, where $s$ is the scale factor which is set in our research at the fixed value of 1 .

Information about times $T_{1}$ and $T_{2}$ in the lateral plane for the aircraft 1 and 2 is also included in the set of predictor variables. Table 1 lists all 12 variables along with their ranges, where the negative values reflect the positions of aircraft in the radar coordinate system.
Table 1. Predictor variables and ranges

\begin{tabular}{llrrl}
\hline Variable & Notation & Min & Max & Units \\
\hline$x_{1}$ & $d_{x}$ & -48.11 & 51.70 & $\mathrm{nmi}$ \\
$x_{2}$ & $d_{y}$ & -52.93 & 35.78 & $\mathrm{nmi}$ \\
$x_{3}$ & $d_{z}$ & -10297 & 8760 & $\mathrm{ft}$ \\
$x_{4}$ & $d$ & 2.40 & 10297.06 & $\mathrm{ft}$ \\
$x_{5}$ & $v_{x, 1}$ & -691 & 584 & $\mathrm{kn}$ \\
$x_{6}$ & $v_{y, 1}$ & -806 & 473 & $\mathrm{kn}$ \\
$x_{7}$ & $v_{z, 1}$ & -83.10 & 96.66 & $\mathrm{kn}$ \\
$x_{8}$ & $v_{x, 2}$ & -444 & 599 & $\mathrm{kn}$ \\
$x_{9}$ & $v_{y, 2}$ & -527 & 426 & $\mathrm{kn}$ \\
$x_{10}$ & $v_{z, 2}$ & -95.05 & 11.53 & $\mathrm{kn}$ \\
$x_{11}$ & $T_{1}$ & 0 & 9 & $\mathrm{~s}$ \\
$x_{12}$ & $T_{2}$ & 0 & 9 & $\mathrm{~s}$ \\
\hline
\end{tabular}

In our experiments we used 2,526 radar cycles that represent traces of 66 aircraft pairs that were landing or taking off at Heathrow. These traces were selected because of approaching a critical distance, so that an average alert rate was $19.7 \%$. The number of cycles in a trace was dependent on the aircraft velocities and, on average, was around 40.

\section{Simulation of Alert Probabilities}

As discussed in Section 1, the "ground truth" in alert systems is not available, and probabilities of alerts have to be simulated in a given environment. In this section we describe how alert probabilities are calculated within our approach by using geometrical extrapolation of the CPA defined by distance $D$ and time $T$, defined in [2728]. Let a function $f_{A}(D, T)$ denote the extrapolated proximity between aircraft on a dangerous course. The function $f_{A}$ allows us to estimate the probability of alert $P_{A}$ for each given cycle in the trajectory. Note that this function does not handle other factors which determine aircraft trajectories, and so it cannot fully explain the behaviour of the STCA system on given flight data.

In our approach, the function $f_{A}$ is built from the recorded flight tracks represented by the distances $D$ and time $T$ which are calculated for the CPA of aircraft at each cycle of the recorded tracks. The flight data include the STCA outcomes for normal (0) and alert (1) cycles. Alert probabilities $P_{A}$ are then calculated from the function $f_{A}(D, T)$ for given $D$ and $T$. The probabilities $P_{A}$ are used for testing the ability of an alert prediction method to distinguish the uncertainty 
in the predicted outcomes between two groups, certain and uncertain.

A system for alert predictions is designed to be able to estimate the uncertainty in the predictions within the probabilistic framework. This ability in our approach is evaluated on the recorded flight data by analysing deviations, $\sigma$, of the posterior probabilities of alert.

Let us consider the flight data that include $n$ cycles made available for modelling of an STCA system and an estimator capable of predicting the probability density and estimating $\sigma_{i}$ for each cycle $i=1, \ldots, n$. Having divided the flight data into training and test data sets, we can design an ensemble estimator on the training data and then calculate the standard deviation $\sigma_{i}$ for all cycles included in the test data. We can then consider a threshold deviation $\sigma_{0}$ which divides the predictions made by the estimator into two groups: one with $\sigma_{i} \leq \sigma_{0}$ and the other with $\sigma_{j}>\sigma_{0}, i \in \mathbf{I}_{1}, j \in \mathbf{I}_{2}$, where $\mathbf{I}_{1}$ and $\mathbf{I}_{2}$ are the indexes of cycles in the two groups, respectively. The $\sigma_{0}$ is a threshold which lies between $\min \left(\left\{\sigma_{i}\right\}_{1}^{n}\right)$ and $\max \left(\left\{\sigma_{i}\right\}_{1}^{n}\right)$. The predictions in the first group have a smaller $\sigma$ than those in the second group, and thus the uncertainties in the alert probabilities predicted for cycles assigned to the first group are smaller than those assigned to the second group.

We can then verify whether the difference between the two groups of cycles, in terms of the extrapolated alert probabilities $P_{A}$, is significant for a given threshold $\sigma_{0}$. If the difference is found to be significant for a threshold $\sigma_{0}$, we can then accept that predictions in group $\mathbf{I}_{1}$ are certain and, by contrast, predictions in group $\mathbf{I}_{2}$ are uncertain. In our experiments we found that the model $f_{A}$, built on the retrospective flight data, explains $0.801 \pm 0.006$ of STCA predictions within 3fold cross-validation. Appendix A describes the details of the geometric extrapolation of aircraft trajectories.

\section{Bayesian Averaging over Decision Tree Models}

This section provides details of MCMC implementation of Bayesian averaging over DT models. DTs are known as hierarchical models consisting of splitting and terminal nodes. DT models are said to be binary if the splitting nodes divide data points into two disjoint subsets. The terminal node assigns an input to one of the possible classes, the probability of which is dominant [58]. For interpretation purposes, the single DT which provides the Maximum a Posteriori probability could be selected from a set of DT models that were collected for averaging [64].

\subsection{Markov chain Monte Carlo algorithm}

Except for trivial cases the Bayesian methodology of averaging over DTs can be feasibly implemented with MCMC approximation. For the approximation, the parameters, $\theta$, of a DT candidate are drawn from the given proposal distributions. A candidate is accepted or rejected according to Bayes rule calculated on the given data $\mathbf{D}$. Given the $m$-dimensional input vector $x$ which represent the flight parameters described in Table 1 . data $\mathbf{D}$, and model parameters $\theta$, the predictive posterior distribution $p(y \mid x, \mathbf{D}), y \in\{1, C\}$, is obtained by combining the predictive posterior distribution $p(y \mid x, \theta, \mathbf{D})$ of parameters $\theta^{(i)}$ conditioned on data $\mathbf{D}$, where $C$ is the number of classes. Full details of this process are given in our previous work [37].

In practice, information about the posterior $p(\theta \mid \mathbf{D})$ is often limited. In such cases the MCMC approximation is achieved with a Metropolis-Hastings sampler, which explores the posterior distribution by making random proposals, see e.g. [65[66].

When DT models are grown, their dimensionality (or number of nodes) varies. The Reversible Jump (RJ) extension of MCMC makes possible the approximation over such models [67]. Given priors and a sufficient number of samples, the RJ MCMC technique explores the posterior distribution and takes samples of model parameters.

The exploration of DT models of variable size has been efficiently made by using the following moves:

Birth moves randomly split the data points falling in one of the terminal nodes by a new splitting node with a variable and rule drawn from the corresponding priors.

Death moves randomly pick a splitting node with two terminal nodes and assign it as a single terminal with the united data points.

Change-split moves randomly pick a splitting node and assign it a new splitting variable and rule drawn from the corresponding priors.

Change-rule moves randomly pick a splitting node and assign it a new rule drawn from a given prior.

The first two moves lead to a change in the dimensionality of parameters. The other moves explore the distribution within the current dimensionality. In particular, the change-split move makes "large" jumps which potentially increase the chance of sampling from a maximal posterior. By contrast, the change-rule move makes "local" jumps in order to explore the details of an area of interest.

As the birth and death moves change the dimensionality, Bayes' rule includes a ratio to achieve the 
condition for reversibility of Markov Chain. Details of the algorithm are given in [37].

There are two phases of MCMC simulation for Bayesian learning of DT models. At the first, so-called burn-in, phase the MCMC explores the parameters of a DT model in order to find areas with a high likelihood on the given data set. At the second, so-called post burn-in phase, samples of a DT model are collected for BMA. The most accurate results of BMA are achieved when prior information on DT models is available [6869].

Within the Bayesian method, the best accuracy of approximation of predictive density is achieved when samples collected during the post burn-in phase are diverse. However, in practice the desired diversity of DT models cannot be achieved in reasonable computing time when prior information on the models is absent or incomplete [69].

In general, the likelihood distribution of the model can have multiple modes, which limits the ability of MCMC to explore the full posterior distribution, as described, e.g., in [34]. However, our research is not specifically focused on this problem. Instead we consider the limitations of MCMC sampling of DT models, which are caused by their hierarchical structures, as discussed in [69]. These limitations lead to poor mixing of DT models because of excessive number of nodes in the grown DT models. Consequently, the ensemble of DT models collected during the MCMC sampling will underperform, as shown in our work [70].

\subsection{RJ MCMC sampler}

When the Metropolis-Hastings algorithm makes a birth move, a terminal node with $n$ data points $\left\{x^{(i)}\right\}_{1}^{n}$ is proposed to be a new splitting node with a variable $j \sim U(1, m)$ and a threshold $q^{\prime} \sim f(x)$, where $f(x)$ is the distribution function of data $\left\{x^{(i)}\right\}_{i}^{n}$. The function $f(x)$ is required to set a prior on the birth move, as described in Section 5.1 As the knowledge of $f(x)$ for each splitting node is limited, a uniform prior, $U(a, b)$, is used, where $(a, b)$ is an interval in which a proposal $q^{\prime}$ is expected.

A change move, that is applied to a DT splitting node, redistributes the data points that fall into the downstream nodes, and therefore can produce a splitting node in which one of two branches contains fewer data points than $p_{\text {min }}$. This condition can be written as

$$
\min \left(n_{\text {left }}^{(i)}, n_{\text {right }}^{(i)}\right)<p_{\text {min }},
$$

where $n_{\text {left }}^{(i)}$ and $n_{\text {right }}^{(i)}$ are the numbers of data points in the left and right branches of the $i$ th splitting node.

If the prior on the number of splitting nodes is given properly, most samples are expected to be drawn from the posterior that is related to areas of interest. If such a prior is unavailable, a DT model will grow excessively and most of the samples will be drawn from posterior distributions that are calculated for oversized DT models. As a result, the estimates of the predictive distribution will be biased, see e.g. [69].

In practice, priors on DT structures are often unavailable, and the MCMC sampler cannot efficiently control DT structures, which leads to poor mixing. However, the DT structure can be better controlled with a sweeping strategy of the MCMC approximation as proposed in [70]. The main idea behind this strategy is to assign the prior probability of splitting DT nodes dependent on the range of values within which the size of a new data partition will exceed $2 p_{\min }$.

This prior is adapted to the range of a data partition. The new splitting threshold $q^{\prime}$ proposed for variable $j$ and partition $i$ is drawn from a uniform distribution: $q^{\prime} \sim U(a, b)$, where $(a, b)$ is the interval of variable $x_{j}$ at node $i$ : $a=\min \left(x_{j}\right)$ and $b=\max \left(x_{j}\right)$.

When the change move is applied to a node that is close to the DT root, distributions of data points in its terminal nodes can be greatly changed, and one or more terminal nodes can contain fewer data points than $p_{\text {min }}$. If there is one such node, this node is swept from the DT and the move is counted as a death move. In cases when there is more than one such node, the move is deemed unavailable according to the MCMC strategy [70].

Our proposed MCMC strategy differs from that described in [70] in the following aspect. Making a birth or change move, the MCMC algorithm proposes a new parameter $q^{\prime}$ which is assigned within an interval $(a, b)$. This interval is estimated from the data samples $\left\{x^{(i)}\right\}_{1}^{n}$ that fall into the DT node assigned for the move. Thus the knowledge of $(a, b)$ excludes assigning a proposal $q^{\prime}$ outside of this interval. This strategy satisfies the condition for reversibility of a Markov Chain, which is needed in order to provide an equal probability of assigning the reverse $q$. As a result, the efficiency of MCMC, in terms of acceptance rate and details of exploring posterior parameters, is expected to be increased. The detailed exploration is critically important in order to achieve the model diversity required for accurate approximation of the predictive density.

The next section outlines pseudocode of the proposed adaptive strategy. 


\subsection{Pseudocode of adaptive strategy}

Algorithm 11 outlines the main steps of the adaptive strategy of assigning proposals. The algorithm receives data points $\left\{x^{(i)}\right\}_{1}^{n}$ that are required to be split by a given node, as well as $p_{\min }$ which defines the minimal number of data points allowed in a node's branch. The change move is applied to a DT split node to update its current threshold $q$. Having received the data points, the algorithm finds the interval $(a, b)$ which is required to avoid the condition in Eq. 1. The data $\left\{x^{(i)}\right\}_{1}^{n}$ are sorted into ascending order in line 3 in order to find an index $i$ of the current threshold $q$ in line 4. Indexes $i_{1}$ and $i_{2}$ are counted within the allowed range and then $x_{\min }$ and $x_{\max }$ are assigned in lines 5 and 6 . The parameter $s$ defines the range of a uniform proposal distribution function $g\left(q^{\prime} \mid q\right)=U(q-s, q+s)$ within which a proposal $q^{\prime}$ is drawn in line 8 .

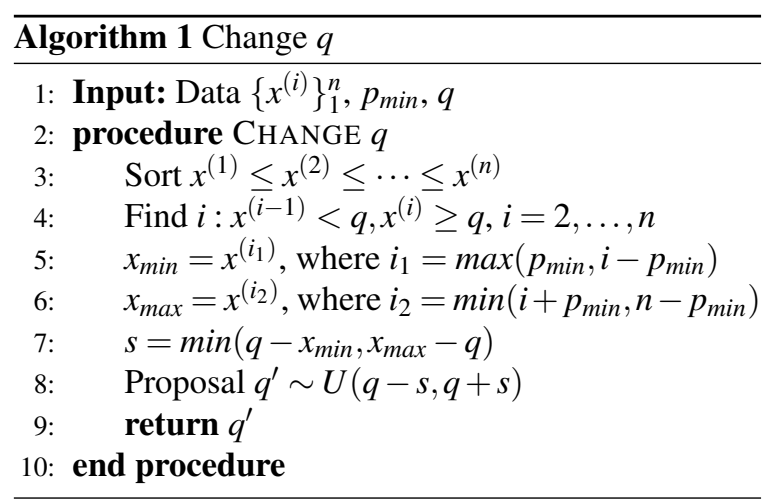

\section{Experiments}

In this section we describe experiments with the proposed Bayesian method on real STCA data. The aim of these experiments was to verify the ability of the proposed method to model outcomes of the STCA system with a reasonable accuracy. In our experiments we aim to find patterns which make uncertain contribution to the prediction. This is required for finding areas in which the accuracy of alert systems can be improved.

\subsection{Experimental settings}

In our experiments STCA probability is predicted for each cycle of an aircraft pair trajectory within the proposed Bayesian framework described in Section 5 . The cycles are represented by the input variables $x_{1}, \ldots, x_{12}$ described in Section 3.1. The Bayesian method esti-
Table 2. Flight Data statistics.

\begin{tabular}{lr}
\hline Characteristic & Value \\
\hline Number of encounters & 66 \\
Total number of cycles & 2526 \\
Average number of radar cycles per trace & 40 \\
Alert rate, \% & 19.7 \\
\hline
\end{tabular}

mates a predictive probability density of an alert for a given cycle. Estimates of the probability density are then used for evaluating the uncertainty of the prediction. If the uncertainty in terms of deviation of the predictive probability density is high, the prediction is said to be "uncertain", otherwise the prediction is said to be "certain". For estimating predictive posterior densities, the Bayesian method employs DT models built on the recorded flight data. DT models grown on these data have different sizes and so the Bayesian method uses RJ MCMC to handle variable dimensionality of model parameters. Reversible Jumps are made by the MCMC sampler outlined in Section 5 by proposing the parameter $q^{\prime}$ for the moves which change dimensionality of the DT model. The proposed changes can be rejected or accepted. Eq. 1 defines a condition when DT node $i$ is unavailable for a proposed move. Algorithm 1 outlines moves that are more likely to be accepted and thus the efficiency of the MCMC sampler will be improved. This condition defines when a DT node can further split the data into smaller subsets.

DT models outlined in Section 2 are used for STCA prediction as follows. The input variables which represent the flight data at a given cycle feed the DT models accepted by the MCMC algorithm. The average over outcomes of these models is the predicted probability of alert at the given cycle. The model outcomes represent a predictive probability distribution of the alert, which is required in order to estimate the uncertainty in predictions. As discussed DT models are easy-to-interpret and for this purposes the Maximum a Posterior model can be selected. An example of the interpretation of DT models for predicting alerts is provided in Section 6.4

The experiments were run within 10-fold cross validation so that each fold contains 59 aircraft traces, which were selected for training, and the remaining 7 for testing the accuracy of alert detection. Table 2 summarises the information about the flight data, described in Section 3.1, which were used in our experiments. 
An advantage of using DT models is that these models are applied "off-the shelf" as noted in [52], and so the BMA method does not require many settings, as discussed in 6869].

The proposed Bayesian method was run with a uniform prior on DT models as there was no information about possible DT structures. The minimal number $p_{\min }$ was set equal to 5 . The proposal probabilities for the birth, death, change-split and change-rules were set to $0.1,0.1,0.2$, and 0.6 , respectively. The first two probabilities, the birth and death moves set to 0.1 , enabled the MCMC sampler to explore DT configurations with a reasonable intensity during the burnin phase. Typically, larger DT configurations require more intensive proposals of the birth and death moves, see e.g. [37].

Setting the probability of the change-split to 0.2 enabled the MCMC algorithm to make the "large jumps" that increase a probability of exploring all areas of interest and avoiding oversampling from areas of local maxima. The remaining proposal probability of 0.6 is assigned to the change-rule to enable the sampler algorithm to explore details of the posterior distribution of DT model parameters of a current configuration. We found that with the given priors the above proposal probabilities provided the best performance of the Bayesian method with a reasonable efficiency of MCMC sampling, which is achieved when an acceptance rate ranges between 0.25 and 0.7 , according to [34].

The number of burn-in and samples was set to 100,000 in order to achieve a stationary Markov chain. In order to collect sufficient posterior samples to achieve a desired approximation accuracy, the number of post burn-in samples was set to be 10,000. Markov chains generate correlated samples, and so the generated samples were decorrelated in order to obtain an i.i.d. sequence by drawing each 10th sample in the post burn-in phase. The proposal variance was set to 4.0 to achieve an acceptance rate of updating the Markov chain around 0.4, which is within the range of efficient MCMC sampling. With these settings, the Bayesian performance within the 10-fold cross-validation was $82.1 \% \pm \sigma$, where $\sigma=5.1 \%$ is the standard deviation.

In the burn-in phase the Markov chain started with log a low likelihood value around -1000 , converging to a higher value that oscillates around -175. In the post burn-in phase the log likelihood oscillated between 200 and -150 . The lower plots show that the average number of DT nodes was around 46.

\subsection{Experimental settings for Random Forest}

RF, as an ensemble method discussed in Section 2, can approximate posterior predictive probability distributions. In our experiments we applied this technique to the flight data with the following parameters. The number of DT models (or ensemble size) was 5,000. The bootstrap sampling rate (or fraction of input data to sample) was set to 0.7 . The number of predictors to be randomly selected for DT splits was 10 out of the 12 described in Table 1 These parameters have enabled the RF to achieve a highest prediction accuracy of $83.2 \%$ and so approximate a probability distribution of interest.

\subsection{Analysis of uncertainty in alert predictions}

This section describes the experiments aimed at evaluating the uncertainty in STCA predictions on the flight data described in Section 3.1 The data are represented by the predictor variables that characterise the flight trajectories of aircraft pairs. The tracks were selected with alert cycles that were recorded nearest to a closet point of approaching. In the given airport environment the number of such tracks was very small. The total number of cycles was 2,526, and each cycle has a label $\mathrm{A}=1$ (alert) or $\mathrm{A}=0$ (normal). The experiments were run to compare the proposed Bayesian and existing RF methods in terms of accuracy of estimating the uncertainty of predictions made by the STCA system on the given flight data.

Let us consider track 2 shown on Fig. 5 and estimate the posterior predictive probability densities of alert for each cycle by using the proposed (BDT) and existing RF methods. The related experimental settings were discussed in Sections 6.1 and 6.2. Fig. 5 shows this track including 42 cycles in which cycles 21 to 33 were detected as alert (shown in Red), and cycles before 22 and after 33 are normal.

Fig. 2 shows the distances $D_{X Y}$ between aircraft pair A1 and A2 along with the extrapolated probabilities of alerts, $P_{A}$, for cycles 1 to 42 . The probabilities $P_{A}$ increase from cycle 1 , having a drop at cycle 11 as a result of change in flight direction of aircraft A2, shown on Fig. 5. Then between cycles 20 and 31 the probabilities $P_{A}$ reach a maximum around 0.67 , whilst the STCA system has predicted alert events between 21 and 33 cycles. We see that the estimations of $P_{A}$ explain the alert events along the cycles, but important information about uncertainties in the predictions is absent. 
$\mathrm{RF}$ as an ensemble method, discussed in Sections 1 and 2 is capable of approximating the posterior probability densities, providing the predicted probabilities along with the ensemble variances which reflect the uncertainty in predictions. The predicted probabilities, $P_{R F}$, and the ensemble standard deviation, $\sigma$, were calculated for the cycles 1 to 41 and shown as error bars on the third subplot of Fig. 2 The $\sigma$ reflect the uncertainties in predicted probabilities $P_{R F}$. We can see that the values of $\sigma$ remain large between cycles 1 and 20 in the absence of alerts as well as between cycles 21 and 34 in the presence of alerts. The values of $\sigma$ decrease only after the last alert cycle 34 . Thus the RF based estimator fails to indicate the relevant uncertainty level at cycles which precede the alerts.

The posterior probability densities of interest were calculated by the proposed BDT method and are shown in the fourth subplot of Fig. 2 as error bars. We see that the alert probabilities $P_{B D T}$ and the values of $\sigma$ differ from those calculated by the RF estimator. Both the alert probabilities $P_{B D T}$ and $\sigma$ at cycles 1 to 18 remain low, except cycles between 9 and 13 when the aircraft A2 has changed the flight direction. The probabilities $P_{B D T}$ increase to 0.9 during alert cycles between 21 and 33. Thus in comparison with the RF estimator, the BDT provides more accurate information on the predicted alert probabilities and uncertainties. The RF estimator provides predictions variations of which are unreasonably large.

It is interesting to observe in Fig. 2 and Fig. 5 that at cycle 10 the aircraft $\mathrm{A} 2$ changed direction so that the distance of CPA was increased, thus decreasing the probability of conflict. The alert probabilities $P_{R F}$ and $P_{B D T}$ predicted at this cycle are equal to 0.31 . However, at the previous cycle 5 of these probabilities were $P_{R F}=0.23$ and $P_{B D T}=0$ with the $\sigma_{R F}=0.363$ and $\sigma_{B D T}=0.05$, respectively. This shows that the BDT outcomes at cycles 5 and 10 are more realistic than the RF outcomes.

Table 3 shows the standard deviations $\sigma_{R F}$ and $\sigma_{B D T}$ calculated for the cycles $k=5,10, \ldots, 35$ along with the alert probabilities $P_{R F}$ and $P_{B D T}$ and alert labels $A \in\{0,1\}$. It is interesting to note that cycle 20 is normal whilst the next cycle 21 is alert, but the RF estimator predicts the cycle 20 with $P_{R F}=0.5$ and $\sigma_{R F}=0.383$, whilst the BDT gives $P_{B D T}=0.45$ and $\sigma_{R F}=0.451$. Cycle 20 has $A=0$ and the BDT outcome is more accurate than that provided by the RF. For the other cycles the values of $\sigma_{B D T}$ are substantially smaller than $\sigma_{R F}$.

The above results allow us to make the following observations:
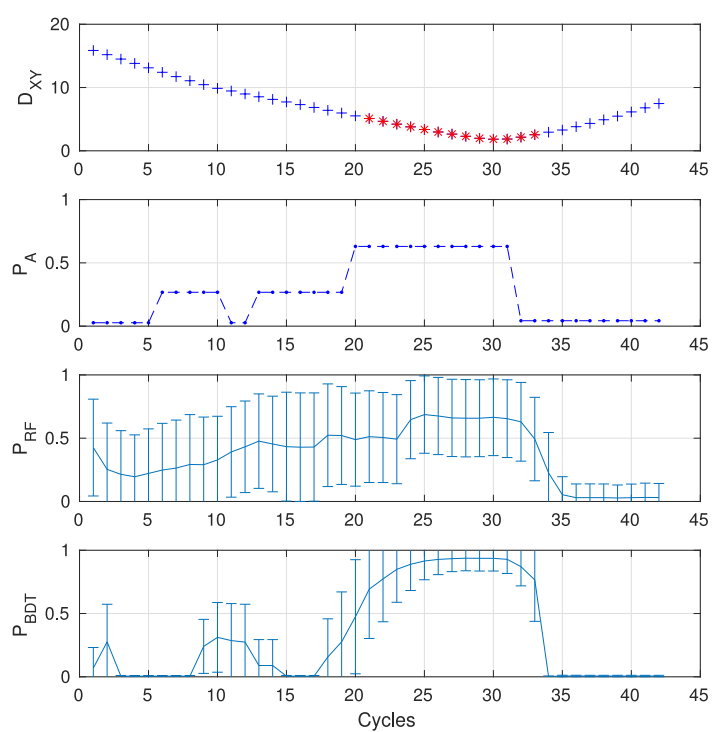

Figure 2. Distances $D_{X Y}$ between aircraft pair A1 and A2 (subplot 1) along with the extrapolated probabilities of alerts $P_{A}$ (subplot 2) over cycles 1 to 42 . Posterior predictive probability densities of alert for cycles 1 to 42 estimated by the existing RF (subplot 3 ) and proposed BDT (subplot 4) methods.

Table 3. Standard deviations $\sigma$ for RF and BDT methods

\begin{tabular}{cccccc}
\hline$k$ & $\sigma_{R F}$ & $\sigma_{B D T}$ & $P_{R F}$ & $P_{B D T}$ & $A$ \\
\hline 5 & 0.363 & 0.005 & 0.23 & 0.00 & 0 \\
10 & 0.352 & 0.275 & 0.31 & 0.31 & 0 \\
15 & 0.435 & 0.005 & 0.45 & 0.00 & 0 \\
20 & 0.383 & 0.451 & 0.50 & 0.47 & 0 \\
25 & 0.310 & 0.148 & 0.67 & 0.91 & 1 \\
30 & 0.295 & 0.100 & 0.67 & 0.94 & 1 \\
35 & 0.129 & 0.006 & 0.05 & 0.01 & 0 \\
\hline
\end{tabular}

(1) Variations in model outcomes included in an ensemble estimator reflect the ability of the estimator to approximate the posterior probability density of alert for a cycle.

(2) The standard deviation $\sigma$ of the posterior probability density estimated for a cycle reflects the uncertainty in the prediction.

Given a threshold deviation $\sigma_{0}$, we can find ratios of alert and normal cycles which are assigned by the RF and BDT estimators to the certain group. Fig 3 shows these ratios over the thresholds $\sigma_{0}$. The triangles and circles denote that the differences between the certain and uncertain groups are significant in terms of the extrapolated probabilities $P_{A}$. The differences are 

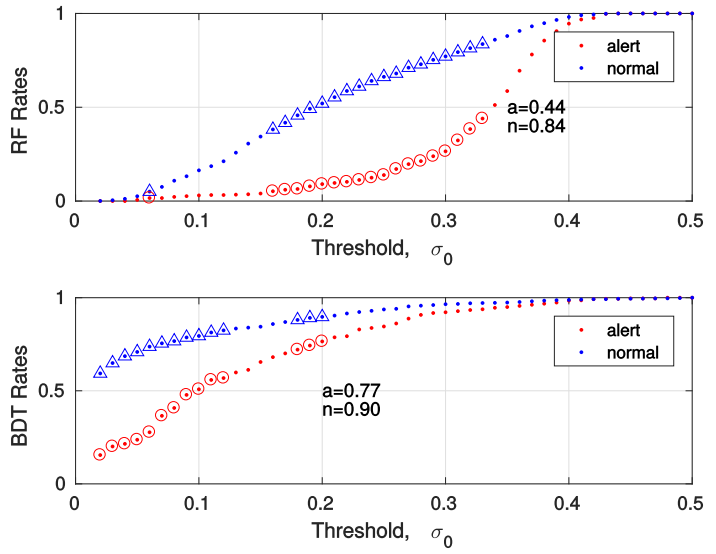

Figure 3. Ratios of alert (Red circles) and normal (Blue triangles) cycles assigned to the certain group by the RF and proposed BDT estimators: $a$ and $n$ are the ratios of alert and normal cycles, respectively.

verified with a 2 -sample Kolmogorov-Smirnov test at a significance level $\alpha<0.05$.

The maximum rates of the certain alert and normal cycles, which are obtained with the RF and BDT estimators, are $a_{R F}=0.44, n_{R F}=0.84$ and $a_{B D T}=$ $0.77, n_{B D T}=0.90$, respectively. The Wilcoxon signed rank test shows that in comparison with RF the proposed BDT method provides a statistically significant increase, $p<2 \cdot 10^{-5}$, in the numbers of certain predictions.

Cycles assigned to the uncertain group can be visualised on a plane of distances $D$ and time $T$ of CPA, calculated for given flight data. The scattering of cycles which have been assigned to the uncertain group by the RF and BDT estimators is plotted in Fig. 4 on the right and left plots, respectively.

On these plots we can observe that the normal cycles (in Blue) are scattered more widely and intensively for the RF method than those assigned by the BDT method. The alert predictions (in Red) are also scattered more intensively for the RF method. This means that the RF method providing a comparable prediction accuracy tends to overestimate the uncertainty in both the alert and normal predictions.

\subsection{Interpretation of alert patterns}

Following [7172], we would like to consider how models developed for alert predictions can be interpreted. This section provides an example of using DT models for predicting patterns of interest. According to our approach Fig. 2 shows the probabilities of alerts for the RF and BDT methods. In this Figure we can
Table 4. A subtree predicting alert probability at cycle 2 .

\begin{tabular}{rrrrrr}
\hline$i$ & $v_{i}$ & $q_{i}$ & $p_{1}$ & $p_{2}$ & $x_{i}$ \\
\hline 1 & 4 & 3543.75 & & 0.00 & 1807.06 \\
2 & 7 & 11.78 & & & 13.25 \\
3 & 3 & -1678.53 & & & -1807.00 \\
4 & 9 & -247.36 & 0.00 & & 273.00 \\
5 & 10 & 0.00 & 0.04 & & 26.68 \\
6 & 8 & -315.03 & 1.00 & & 68.00 \\
7 & 4 & 2276.87 & 0.77 & 0.09 & 1807.06 \\
\hline
\end{tabular}

observe unexpectedly high alert probabilities $P_{R F}$ and $P_{B D T}$ for cycles 1 and 2 , which on average are 0.2 and 0.4 respectively. We can explain these events by the following.

Let us first find the DT models that predict the highest alert probability, which is 0.77 for cycle 2 . The proportion of such models is $9.1 \%$. The high probability predicted by these models is explained by the following. The flight data, represented in Table 1 , include the distance between aircraft pair, $x_{4}$, which makes the determining contribution to the prediction for cycle 2 .

The prediction is made by a subtree path which is shown in Table 4. This table represents the rules $i=1, \ldots, 7$, indexes of input variables $v_{i} \in\{1,12\}$, thresholds $q_{i}$, terminal probabilities $p_{1}$ and $p_{2}$. The subtree tests the rules and assigns the right branch if $x_{i}>q_{i}$ and the left branch if otherwise. The rules 1 and 7 contain the right-branch terminals with probabilities of alert 0.00 and 0.09 . The rules 4 to 7 contain the left-branch terminals with the probabilities of alerts as shown. For the given cycle 2 , the input falls into the left-branch terminal of rule 7 which assigns an alert probability $p_{B D T}=0.77$. The distance $x_{4}=1807.06$ is critically close to the threshold $q=2276.87$, and so the predicted probability remains high.

The above scenario can be used in a similar way for interpretation of other patterns of interest.

\section{Discussion}

Alert systems are designed to predict critical events when the "ground truth" is unavailable and the accuracy of alert predictions cannot be directly evaluated from given data. Uncertainty which exists in the data and models affects the accuracy in such an application as air-traffic conflict avoidance using STCA systems that aim to inform controllers about possible conflicts in an intensive airport traffic environment. The uncer- 

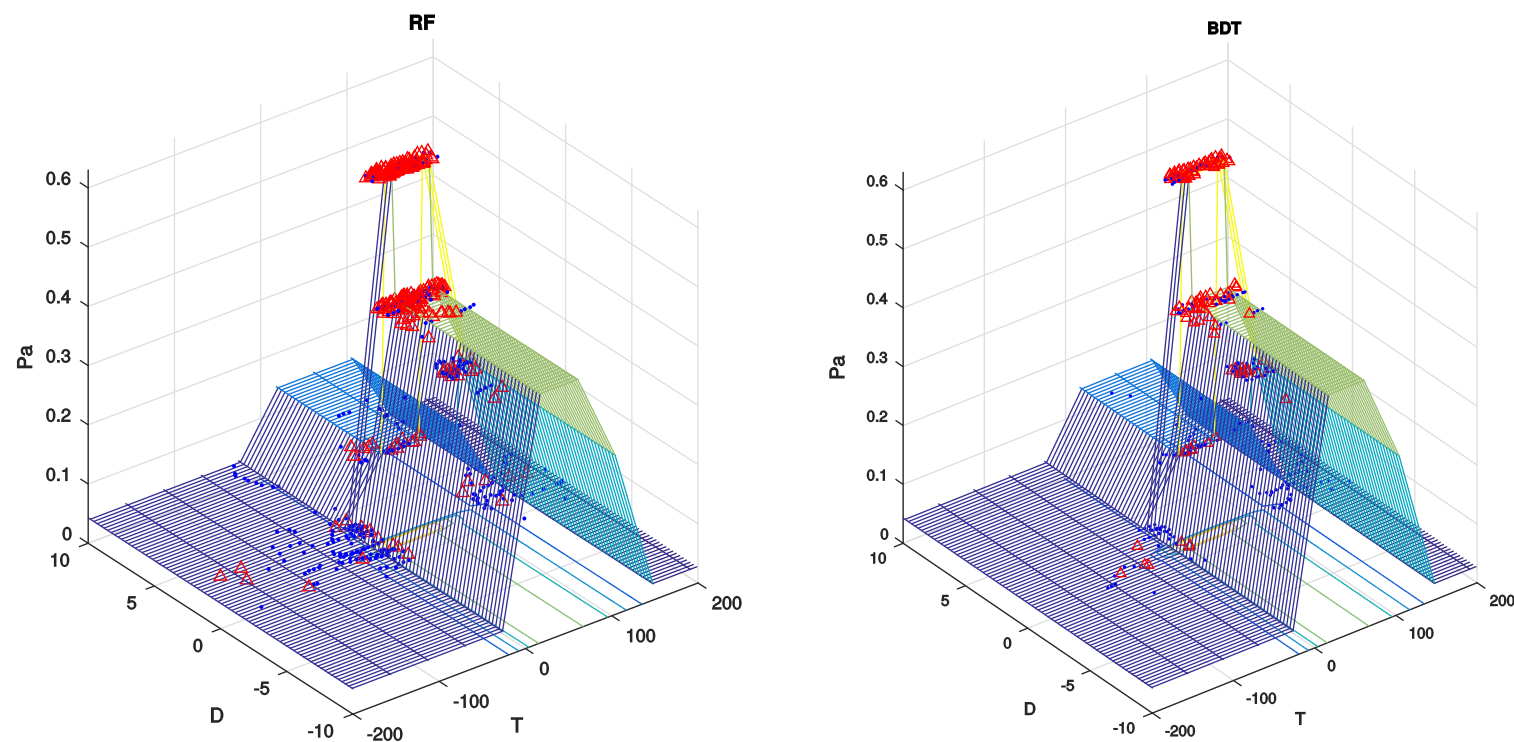

Figure 4. Cycles assigned to the uncertain group spread over distances $D$ and time $T$ of CPA. The vertical axis shows the extrapolated alert probabilities $P_{A}$. Alerts are shown in Red, and normal cycles are in Blue. Predictions are assigned to the uncertain group by the RF and BDT methods on the left and right plots, respectively.

tainty affects accuracy of predicting the coordinates of aircraft and increases errors of alert prediction. Therefore it is critically important to find new insights into areas in which alert systems can be improved in the absence of the ground truth.

In our study we used a probabilistic approach to learn a model of an STCA system from recorded flight data, and expect that such an approach is able to provide realistic insights into the uncertainty in alert predictions. The accurate identification of uncertain patterns is required in order to find ways of improving the accuracy of alert detection. Existing methods, outlined in Sections 1 and 2, are often based on unrealistic assumptions which cannot be widely accepted for analysing the uncertainty in alert predictions.

In our previous work, outlined in Section 1 more realistic results have been achieved within a Bayesian framework employing a new MCMC strategy of averaging over Decision Tree models. In this study we extend the Bayesian method, outlined in Section 5, to address the problems of finding the uncertain patterns in alert predictions.

In our experiments we used Heathrow flight data, described in Section 3.1. which include recorded radar tracks and other flight parameters of aircraft pairs as input to the STCA system. We found in these data alerts which have been predicted at different flight conditions variations of which have exceeded the technical ability of the STCA system to keep the accuracy of alert predictions. The experiments have been run on 66 tracks of aircraft pairs with a high rate of alerts which were rare in the normal operation, so that in total the 2,526 cycles were available. In such a case the sensitivity of alert detection will be higher than that estimated on the real flight data. This however can be effectively managed by using an approach using DT ensembles, as described in [73].

In addition to the core predictors listed in Table 1 the current STCA systems use derived predictors. The core predictors can be extended with, e.g., outcomes of filters, as described in the guidelines [74]. The set of predictors in our approach is not limited by the core variables listed in Table 1 , and so new features can be added.

Following [54] we estimated the uncertainty as variance in posterior predictive probability distributions of alerts. This allowed us to identify cycles with large and small uncertainties for a given variance threshold. The uncertain cycles were significantly different from the certain cycles in terms of probabilities of alerts, which were predicted by using the distances and time of CPA described in Section 4 Under certain conditions, the linear trajectory extrapolation of flight parameters of an aircraft pair approaching each other provides realistic estimates of the distance $D$ and time $T$ of CPA.

The proposed Bayesian method has provided a sufficient accuracy of STCA modelling on the test 
flight data, which allowed us to estimate variances in posterior probability densities of alerts and then find cycles with high and low uncertainties which were assigned to two groups. We have shown that, given a threshold variance, the proposed Bayesian method was able to identify the two groups of patterns in which the contribution to alert prediction is significantly different.

The method has been compared with bootstrap aggregation and demonstrated a statistically significant $\left(p<2 \cdot 10^{-5}\right)$ increase in the numbers of certain predictions. It is interesting to note that the improvement in the accuracy of estimating the uncertainty can be explained by the important property of Markov chains used to explore model parameters, which allows the MCMC sampler to quantify the uncertainty more accurately than a technique based on bootstrap aggregation.

As discussed in Section 1, the improvement of operational characteristics of an STCA system is of crucial importance because uncertainties existing in flight data and the system affect the accuracy of alert predictions. In order to analyse factors which affect the results, the predictions that are made at high uncertainty on the given data have to be identified and analysed. The proposed method provides reliable estimates of predictive posterior density for each prediction in the following way. Patterns of interest, which are identified as uncertain by the method, are represented by DT models. Each predicted outcome can be therefore interpreted by a decision tree, that has the maximum posterior probability. Such a tree consists of $k=1, \ldots, k_{\max }$ splitting nodes, where $k_{\max }$ is the maximal number of nodes in the DT model. A tree model includes the input variables $\left\{x_{i}\right\}_{1}^{k}$ and thresholds $\left\{q_{i}\right\}_{1}^{k}$. The predicted outcome therefore can be explained by the input variables that make determining contribution. An example of such an interpretation has been given in Section 6.4 .

\section{Conclusions and Future Work}

A new approach has been proposed to model alert systems on data which cannot include the "ground truth". In such applications, existing methods cannot provide realistic insights into posterior predictive distributions which are required for analysis. In our experiments with Heathrow flight data including radar tracks of aircraft pairs, we have observed that alerts were predicted under different flight parameters, variations in which often affect the ability of STCA system to maintain accuracy of alert prediction.

The proposed Bayesian method has provided the accuracy of modelling the STCA on the flight data, sufficient for analysing the uncertainties in alert predictions. Estimating the variance in posterior predictive probability distributions of alerts, we have identified cycles that have large uncertainty. The identified uncertain cycles were significantly different from the certain cycles in terms of probabilities of alerts which were predicted using the distances and time of Closest Point of Approach. The proposed method has been compared with bootstrap aggregation and demonstrated a statistically significant increase $(p<$ $2 \cdot 10^{-5}$ ) in the numbers of certain predictions.

In our experiments the proposed approach has realistically estimated the uncertainty of alerts and thus can be used for modelling of alarm systems with the aim of optimisation. We conclude that the Bayesian method is capable of delivering tractable probabilistic information about alerts and thus will be essential for designing alert systems and other critical applications in which the ground truth is unavailable.

Our research has been mainly focused on the reliable analysis of uncertainty in alert predictions. Several questions have not been resolved and so can be addressed in future work. First we think that nonlinear extrapolation of the distance and time to the closest point of approach will provide more realistic calculation of probability of alert $P_{A}$. Inclusion of vertical separation in the extrapolation can also improve the accuracy of alert prediction. Another question which can be also addressed is related to scalability of the method in part of additional information existing in STCA systems, as discussed, e.g., in [74]. It will be undoubtedly interesting to extend the method with the ability of capturing trajectory dynamics, provided within a rigorous framework for dynamic Bayesian networks [36 75], whilst the use of Bayesian data fusion discussed in [76] seems to be also attractive for the future work.

\section{A. Time and Distance to Closest point of Approach}

Based on predicted positions and velocities of an aircraft pair, the alert system generates an alarm if the predicted distance between the aircraft becomes critical. The uncertainty in the data affects the accuracy of alert detection. Besides, alert predictions are influenced by variations in the flight parameters of air- 
craft such as distance and velocities. The distribution of alert events over distances can be estimated from the recorded flight data in order to find a compromise between the false and missed alert rates. The compromise is consequently dependent on factors representing the flight data [28]. In particular, the relative velocity with which the aircraft are approaching is one such factor [30].

According to [27], distance and time of closet point of approach (CPA) is estimated by using linear extrapolation for each aircraft pair in the $x y$ coordinates. This method assumes the flight parameters of the pair will not be changed in the immediate future [46].

Given an aircraft pair A1 and A2 with positions $\left(x_{1}, y_{1}\right)$ and $\left(x_{2}, y_{2}\right)$ in the $x y$-axes, the distance between the aircraft is

$$
S=\sqrt{\left(x_{2}-x_{1}\right)^{2}+\left(y_{2}-y_{1}\right)^{2}} .
$$

The angle between the aircraft is defined as

$$
Q_{s}=\arctan \left(\frac{y_{2}-y_{1}}{x_{2}-x_{1}}\right) .
$$

The relative velocity $V$ with which the aircraft are approaching is

$$
V=\sqrt{\left(v_{x, 2}-v_{x, 1}\right)^{2}+\left(v_{y, 2}-v_{y, 1}\right)^{2}},
$$

where $v_{x}$ and $v_{y}$ are the velocities of the aircraft along the $x$ and $y$ axes, respectively.

The angle between the velocity vectors of the aircraft is

$$
Q_{v}=\arctan \left(\frac{v_{y, 2}-v_{y, 1}}{v_{x, 2}-v_{x, 1}}\right)
$$

Having assumed that the above velocity $V$ is unchanged in the immediate future, the aircraft will cross the CPA at the minimal distance $D$ which is defined according to the sine rule as:

$$
D=S \sin \left(Q_{s}-Q_{v}\right)
$$

According to this rule, the minimal $D$ is the perpendicular from A1 to the line of the vector $V$. The arrival time at the CPA is

$$
T=\frac{S \cos \left(Q_{s}-Q_{v}\right)}{V} .
$$

Note that after crossing the CPA, the distance $D$ and time $T$ become negative. An alarm is raised if the distance $D_{i}$ and time $T_{i}$ estimated at a cycle $i$ are positive and their absolute values are smaller than the predefined critical values $D_{0}$ and $T_{0}:\left|D_{i}\right|<D_{0} \&$ $\left|T_{i}\right|<T_{0}$.

Fig. 5 plots the distances $S$ and the velocities $V$ for the aircraft A1 and A2 along 42 cycles. The cycles represent track 2 over the $x y$ coordinates for the flight data described in Section 3.1. The system has detected 13 alert cycles denoted by the Red stars.

In particular at cycle 1 the distance $S$ between aircraft A1 and A2 is $15.8 \mathrm{nmi}$. The aircraft have velocities $V_{1}=6.39$ and $V_{2}=7.06$, so that the relative velocity $V=7.06$ The distance vector angle is $Q_{s}=14.8^{\circ}$ whilst the relative velocity vector angle is $Q_{v}=13.1^{\circ}$. At cycle 1 the aircraft will approach the CPA with distance $D$ for this cycle is $0.48 \mathrm{nmi}$ and the time $T=134 \mathrm{~s}$. This time is not critical and so the system does not raise an alarm for cycle 1 .

The first alert is detected at cycle 21 when the estimates $D$ and $T$ are less than the predefined critical values $D_{0}$ and $T_{0}$. The initiated alert sequence ends at cycle 33 as shown on Fig. 5 .

Having undertaken a manoeuvre during the alert cycles, both aircraft have urgently changed flight parameters. Cycle 35 which is two cycles after the last alert is shown in Fig. 5 After crossing the CPA with minimal distance at cycle 30 , the aircraft at cycle 35 arrived at the new positions and the distance was increased to $S=3.28$. At this cycle the aircraft have velocities $V_{1}=6.52$ and $V_{2}=4.25$ so that the relative velocity $V=3.28$ is nearly half that at cycle 1 . After the manoeuvre, the distance angle was significantly increased to $Q_{s}=241^{\circ}$, so that the velocity vector angle increased to $Q_{v}=78.4^{\circ}$. With the new flight parameters the aircraft pair has then diverged, so that both the distance $D=-0.93$ and time $T=-50$ are negative after crossing the CPA.

The above model is based on linear extrapolation and assumptions that flight parameters of the aircraft pair are not changed in the time before crossing the estimated CPA. The model has been extended with velocity acceleration as well as with resolution of vertical conflicts [29]. A number of probabilistic extensions has been undertaken [20].

Fig. 5 illustrates how the above geometrical extrapolation is used for predicting an alert event probability. Section 6.3 provides details and the experimental settings for this technique. 


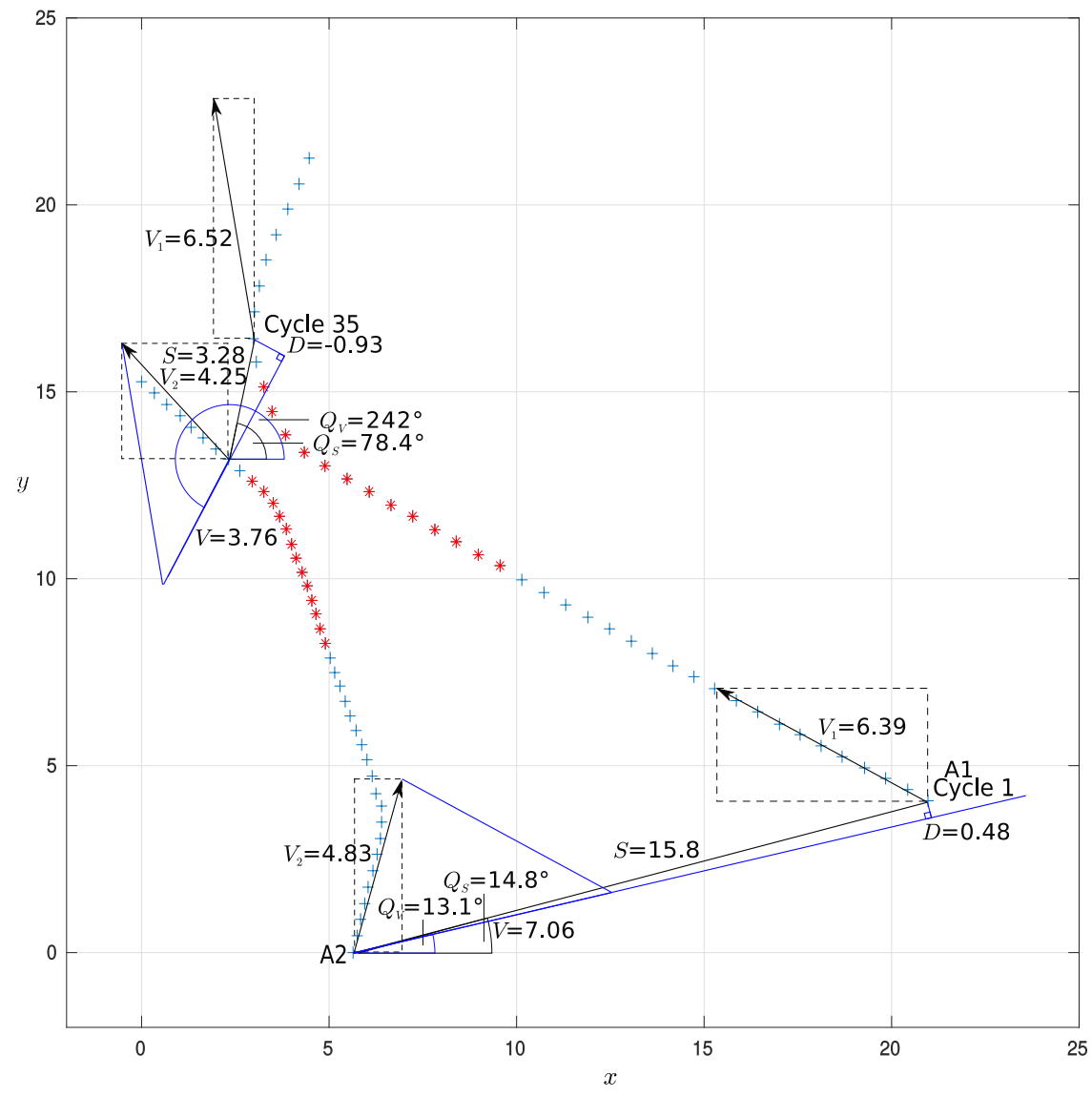

Figure 5. Distances $S$ and the velocities $V$ for the aircraft A1 and A2 along 42 cycles which represent track 2 over the $x y$ coordinates. The 13 alert cycles (in Red) were detected by the STCA system.

\section{B. Supplementary materials}

The STCA data described in Section 3.1 are available at/http://figshare.com/articles/Flight_data/ 5446681

\section{Acknowledgements}

The authors are thankful to all the anonymous reviewers for their constructive comments. This research was largely supported by the UK Engineering and Physical Sciences Research Council (EPSRC) grant GR/R24357/01 "Critical Systems and Data-Driven Technology".

\section{References}

[1] J. Jansson and F. Gustafsson, "A framework and automotive application of collision avoidance decision making," Automatica, vol. 44, no. 9, pp. 2347 - 2351, 2008.
[2] Z. Grande, E. Castillo, E. Mora, and H. K. Lo, "Highway and road probabilistic safety assessment based on bayesian network models," Computer-Aided Civil and Infrastructure Engineering, vol. 32, no. 5, pp. 379-396, 2017.

[3] E. Castillo, Z. Grande, E. Mora, H. K. Lo, and X. Xu, "Complexity reduction and sensitivity analysis in road probabilistic safety assessment bayesian network models," ComputerAided Civil and Infrastructure Engineering, vol. 32, no. 7, pp. 546-561, 2017.

[4] H. A. P. Blom, S. H. Stroeve, and H. H. de Jong, Safety Risk Assessment by Monte Carlo Simulation of Complex Safety Critical Operations. London: Springer London, 2006, pp. 47-67.

[5] T. Lauderdale, "Probabilistic conflict detection for robust detection and resolution," in 12th AIAA Aviation Technology, Integration, and Operations (ATIO) Conference, 2012.

[6] M. J. Kochenderfer, M. W. M. Edwards, L. P. Espindle, J. K. Kuchar, and J. D. Griffith, "Airspace encounter models for estimating collision risk," Journal of Guidance, Control, and Dynamics, vol. 33, no. 2, pp. 487-499, 2010.

[7] W. Harman, "TCAS - a system for preventing midair collisions," The Lincoln Laboratory Journal, vol. 2, p. 437458, 1989.

[8] J. Leandro, A. S. Chen, S. Djordjevi, and D. A. Savi, "Com- 
parison of 1D/1D and 1D/2D coupled (sewer/surface) hydraulic models for urban flood simulation," Journal of $\mathrm{Hy}$ draulic Engineering, vol. 135, no. 6, pp. 495-504, 2009.

[9] A. Kosgodagan-Dalla Torre, T. G. Yeung, O. MoralesNpoles, B. Castanier, J. Maljaars, and W. Courage, "A two-dimension dynamic bayesian network for large-scale degradation modeling with an application to a bridges network," Computer-Aided Civil and Infrastructure Engineering, vol. 32, no. 8, pp. 641-656, 2017.

[10] H.-Q. Mu and K.-V. Yuen, "Ground motion prediction equation development by heterogeneous bayesian learning," Computer-Aided Civil and Infrastructure Engineering, vol. 31, no. 10, pp. 761-776, 2016.

[11] D. Rabuffetti, G. Ravazzani, C. Corbari, and M. Mancini, "Verification of operational Quantitative Discharge Forecast (QDF) for a regional warning system: the AMPHORE case studies in the upper Po River," Natural Hazards and Earth System Science, vol. 8, no. 1, pp. 161-173, Feb. 2008.

[12] B. Thiebes, R. Bell, T. Glade, S. Jäger, J. Mayer, M. Anderson, and L. Holcombe, "Integration of a limit-equilibrium model into a landslide early warning system," Landslides, vol. 11, no. 5, pp. 859-875, Oct 2014.

[13] T. Aldemir, "A survey of dynamic methodologies for probabilistic safety assessment of nuclear power plants," Annals of Nuclear Energy, vol. 52, no. Supplement C, pp. 113 124, 2013, nuclear Reactor Safety Simulation and Uncertainty Analysis.

[14] S. Wager, T. Hastie, and B. Efron, "Confidence Intervals for Random Forests: The Jackknife and the Infinitesimal Jackknife," Journal of Machine Learning Research, vol. 15, pp. 1625-1651, 2013.

[15] J. L. Anderson, "A method for producing and evaluating probabilistic forecasts from ensemble model integrations," Journal of Climate, vol. 9, no. 7, pp. 1518-1530, 1996.

[16] I. Lymperopoulos and J. Lygeros, "Sequential monte carlo methods for multi-aircraft trajectory prediction in air traffic management," International Journal of Adaptive Control and Signal Processing, vol. 24, no. 10, pp. 830-849, 2010.

[17] C. D. Wickens, S. Rice, D. Keller, S. Hutchins, J. Hughes, and K. Clayton, "False alerts in air traffic control conflict alerting system: Is there a "cry wolf" effect?" Human Factors, vol. 51, no. 4, pp. 446-462, 2009.

[18] T. Lauderdale, "The effects of speed uncertainty on a separation assurance algorithm," in 10th AIAA Aviation Technology, Integration, and Operations (ATIO) Conference, 2010.

[19] M. Prandini, J. Hu, J. Lygeros, and S. S., "A probabilistic framework for aircraft conflict detection," IEEE Transactions on Intelligent Transportation Systems, vol. 1(4), pp. 199-220, 2000.

[20] J. P. Chryssanthacopoulos and M. J. Kochenderfer, "Accounting for state uncertainty in collision avoidance," Journal of Guidance, Control, and Dynamics, vol. 34, no. 4, pp. 951960, 2010.

[21] D. M. Asmar, M. J. Kochenderfer, D. M. Asmar, and M. J. Kochenderfer, "Optimized airborne collision avoidance in mixed equipage environments," 2013, performing Organization Report No.

[22] Y. Matsuno and T. Tsuchiya, Probabilistic Conflict Detection in the Presence of Uncertainty. Tokyo: Springer Japan, 2014, pp. 17-33.

[23] J. Morrel, "Fundamental physics of the aircraft collision problem," Bendix Corporation, Tech. Rep., June 1956, tech- nical Memo 465-1016-39.

[24] D. Varon, "The effect of tracking accuracy on short term conflict alert performance," Air Traffic Control Quarterly, vol. 8, no. 3, pp. $173-193,2000$.

[25] L. Surhone, M. Tennoe, and S. Henssonow, Short Term Conflict Alert. Betascript Publishing, 2011.

[26] Z. Mahboubi and M. J. Kochenderfer, "Learning traffic patterns at small airports from flight tracks," IEEE Transaction on Intelligent Transportation Systems, vol. PP, no. 99, pp. 1$10,2016$.

[27] A. Shakarian and A. Haraldsdottir, "Required total system performance and results of a short term conflict alert simulation study," in $4^{\text {th }}$ USA/Europe Air Traffic Management Research and Development Seminar, 2001.

[28] S. Alam, H. Abbass, C. Lokan, M. Ellejmi, and S. Kirby, "Computational red teaming to investigate failure patterns in medium-term conflict detection," in 8th EUROCONTROL Innovative Research Workshop and Exhibition, 2009.

[29] R. A. Paielli and H. Erzberger, "Conflict probability estimation generalized to non-level flight," IEEE Transactions on Intelligent Transportation Systems, vol. 7, no. 3, pp. 195-222, 1999.

[30] H. A. P. Blom and G. J. Bakker, "Conflict probability and incrossing probability in air traffic management," in Proceedings of the 41st IEEE Conference on Decision and Control, vol. 3. IEEE Computer Society, 2002, pp. 2421-2426.

[31] S. H. Stroeve, H. A. Blom, and G. B. Bakker, "Systemic accident risk assessment in air traffic by Monte Carlo simulation," Safety Science, vol. 47, no. 2, pp. $238-249,2009$.

[32] H. A. P. Blom and G. J. Bakker, "Safety evaluation of advanced self-separation under very high en route traffic demand," Journal of Aerospace Information Systems, vol. 12, no. 6, pp. 413-427, 2015.

[33] A. Isaksson, M. Wallman, H. Göransson, and M. G. Gustafsson, "Cross-validation and bootstrapping are unreliable in small sample classification," Pattern Recognition Letters, vol. 29, no. 14, pp. 1960-1965, 2008.

[34] C. Robert and G. Casella, Introducing Monte Carlo Methods With R, ser. Use R. Springer, 2009.

[35] S. Taheri and R. Zarei, "Bayesian system reliability assessment under the vague environment," Applied Soft Computing, vol. 11, no. 2, pp. $1614-1622,2011$.

[36] A. J. Weinert, E. P. Harkleroad, J. D. Griffith, M. W. Edwards, and M. J. Kochenderfer, "Uncorrelated encounter model of the national airspace system version 2.0," MIT Lincoln Laboratory, Lexington, MA, Tech. Rep., August 2013, project Report ATC-404.

[37] V. Schetinin, L. Jakaite, and W. Krzanowski, "Bayesian predictive modelling: Application to aircraft short-term conflict alert system," in Proceedings of the Twelfth UAI Bayesian Modeling Applications Workshop (BMAW 2015) co-located with the 31 st Conference on Uncertainty in Artificial Intelligence (UAI 2015), 2015.

[38] T. Bailey, R. Everson, J. Fieldsend, W. Krzanowski, D. Partridge, and V. Schetinin, "Representing classifier confidence in the safety critical domain an illustration from mortality prediction in trauma cases," Neural Computing and Applications, vol. 16, no. 3, pp. 1-10, 2007.

[39] V. Schetinin, L. Jakaite, J. Jakaitis, and W. Krzanowski, "Bayesian decision trees for predicting survival of patients: A study on the us national trauma data bank," Computer Methods and Programs in Biomedicine, vol. 111, no. 3, pp. $602-$ 
612, 2013.

[40] V. Schetinin, L. Jakaite, and W. J. Krzanowski, "Prediction of survival probabilities with bayesian decision trees," Expert Systems with Applications, vol. 40, no. 14, pp. 5466 - 5476, 2013.

[41] L. Breiman, "Bagging predictors," Machine Learning, vol. 24, no. 2, pp. 123-140, Aug 1996.

[42] N. A. T. Services, "Short Term Conflict Alerts," in London Area Control Centre. National Air Traffic Services, London, 2002.

[43] SKYbrary, "Separation standards," 2016, accessed 14/01/2018. [Online]. Available: http://www.skybrary.aero/ index.php/Separation_Standards

[44] J. E. Kuchar and A. C. Drumm, "The traffic alert and collision avoidance system," Lincoln Laboratory Journal, vol. 16 no. 2, 2007.

[45] M. J. Kochenderfer, J. E. Holland, and J. P. Chryssanthacopoulos, "Next-generation airborne collision avoidance system," Lincoln Laboratory Journal, vol. 19, no. 1, 2012

[46] J. K. Kuchar and L. C. Yang, "A review of conflict detection and resolution modeling methods," IEEE Transactions on Intelligent Transportation Systems, vol. 1, no. 4, pp. 179-189, Dec 2000.

[47] K. P. Murphy, "Dynamic Bayesian networks: representation, inference and learning," Ph.D. dissertation, University of California, Berkeley, 2002.

[48] C. von Essen and D. Giannakopoulou, Analyzing the Next Generation Airborne Collision Avoidance System. Berlin, Heidelberg: Springer Berlin Heidelberg, 2014, pp. 620-635.

[49] B. Efron and R. Tibshirani, "Bootstrap methods for standard errors, confidence intervals, and other measures of statistical accuracy," Statist. Sci., vol. 1, no. 1, pp. 54-75, 021986.

[50] T. Hastie, R. Tibshirani, and J. Friedman, The Elements of Statistical Learning, ser. Springer Series in Statistics. New York, NY, USA: Springer New York Inc., 2008.

[51] T. G. Dietterich, "Ensemble methods in machine learning," in Proceedings of the First International Workshop on Multiple Classifier Systems, ser. MCS '00. London, UK, UK: Springer-Verlag, 2000, pp. 1-15.

[52] L. Breiman, "Random forests," Machine Learning, vol. 45, no. 1, pp. 5-32, Oct 2001

[53] L. Mentch and G. Hooker, "Quantifying uncertainty in random forests via confidence intervals and hypothesis tests," $J$. Mach. Learn. Res., vol. 17, no. 1, pp. 841-881, Jan. 2016.

[54] A. Krogh and J. Vedelsby, "Neural network ensembles, cross validation and active learning," in Proceedings of the 7th International Conference on Neural Information Processing Systems, ser. NIPS'94. Cambridge, MA, USA: MIT Press, 1994, pp. 231-238.

[55] L. Jakaite and V. Schetinin, "Feature selection for Bayesian evaluation of trauma death risk," in The 14th Nordic-Baltic Conference on Biomedical Engineering and Medical Physics. Springer, 2008, pp. 123-126.

[56] V. Schetinin and L. Jakaite, "Classification of newborn EEG maturity with Bayesian averaging over decision trees," Expert Systems with Applications, vol. 39, no. 10, pp. 9340-9347, 2012.

[57] L. Jakaite, V. Schetinin, and C. Maple, "Bayesian assessment of newborn brain maturity from two-channel sleep electroencephalograms," Computational and Mathematical Methods in Medicine, vol. 2012, pp. 1-7, 2012.

[58] L. Breiman, J. Friedman, R. Olshen, and C. Stone, Classifi- cation and Regression Trees. Chapman and Hall, 1984.

[59] W. Buntine, "Learning classification trees," Statistics and Computing, vol. 2, p. 6373, 1998.

[60] T. J. Ross, M. R. Taha, J. J. Kim, and F. Gilfeather, "Logical models for the propagation of disparate information and uncertainty across effectivity trees," Integrated Computer-Aided Engineering, vol. 18, no. 3, pp. 251-264, 2011.

[61] A. Bustillo, M. Grzenda, and B. Macukow, "Interpreting treebased prediction models and their data in machining processes," Integrated Computer-Aided Engineering, vol. 23, no. 4, pp. 349-367, 2016

[62] V. Schetinin, D. Partridge, W. J. Krzanowski, R. M. Everson, J. E. Fieldsend, T. C. Bailey, and A. Hernandez, "Experimental comparison of classification uncertainty for randomised and bayesian decision tree ensembles," in Intelligent Data Engineering and Automated Learning - IDEAL 2004, Z. R. Yang, H. Yin, and R. M. Everson, Eds. Berlin, Heidelberg: Springer Berlin Heidelberg, 2004, pp. 726-732.

[63] V. Schetinin, J. E. Fieldsend, D. Partridge, W. J. Krzanowski, R. M. Everson, T. C. Bailey, and A. Hernandez, "Comparison of the Bayesian and randomized decision tree ensembles within an uncertainty envelope technique," Journal of Mathematical Modelling and Algorithms, vol. 5, pp. 397-416, 2006.

[64] H. Chipman, E. George, and R. McCulloch, "Making sense of a forest of trees," in Symposium on the Interface. Interface Foundation of North America, 1998, pp. 223-230.

[65] C. Robert and G. Casella, Monte Carlo Statistical Methods, ser. Springer Texts in Statistics. Springer, 2004.

[66] W. J. Krzanowski, T. C. Bailey, D. Partridge, J. E. Fieldsend, R. M. Everson, and V. Schetinin, "Confidence in classification: A Bayesian approach," Journal of Classification, vol. 22(2), pp. 199-220, 2006.

[67] P. J. Green, "Reversible jump Markov chain Monte Carlo and Bayesian model determination," Biometrika, vol. 82, pp. 711-732, 1995

[68] H. Chipman, E. George, and R. McCullock, "Bayesian CART model search," Journal of American Statistics, vol. 93, pp. 935-960, 1998.

[69] D. Denison, C. Holmes, B. Mallick, and A. Smith, Bayesian Methods for Nonlinear Classification and Regression. Wiley, 2002.

[70] V. Schetinin, J. E. Fieldsend, D. Partridge, T. J. Coats, W. J. Krzanowski, R. M. Everson, T. C. Bailey, and A. Hernandez, "Confident interpretation of bayesian decision tree ensembles for clinical applications," IEEE Transactions on Information Technology in Biomedicine, vol. 11, no. 3, pp. 312-319, 2007.

[71] E. Castillo, Z. Grande, and A. Calvio, "Bayesian networksbased probabilistic safety analysis for railway lines," Computer-Aided Civil and Infrastructure Engineering, vol. 31, no. 9, pp. 681-700, 2016.

[72] E. Castillo, Z. Grande, E. Mora, X. Xu, and H. K. Lo, "Proactive, backward analysis and learning in road probabilistic bayesian network models," Computer-Aided Civil and Infrastructure Engineering, vol. 32, no. 10, pp. 820-835, 2017.

[73] P. Santos, J. Maudes, and A. Bustillo, "Identifying maximum imbalance in datasets for fault diagnosis of gearboxes," Journal of Intelligent Manufacturing, Jun 2015.

[74] The European Organisation for the Safety of Air Navigation, "EUROCONTROL guidelines for short term conflict alert - part III: Implementation and optimisation examples," 2017, accessed 14/01/2018. [Online]. Available: https: //www.skybrary.aero/bookshelf/books/3751.pdf 
[75] E. Castillo, A. Calvio, Z. Grande, S. Snchez-Cambronero, I. Gallego, A. Rivas, and J. M. Menndez, "A markovianbayesian network for risk analysis of high speed and conventional railway lines integrating human errors," ComputerAided Civil and Infrastructure Engineering, vol. 31, no. 3, pp. 193-218, 2016

[76] F.-C. Chen, M. R. Jahanshahi, R.-T. Wu, and C. Joffe, "A texture-based video processing methodology using bayesian data fusion for autonomous crack detection on metallic surfaces," Computer-Aided Civil and Infrastructure Engineering, vol. 32, no. 4, pp. 271-287, 2017. 